\title{
Error Estimates for the Regularization of Optimal Control Problems with Pointwise Control and State Constraints
}

\author{
S. Cherednichenko and A. Rösch
}

\begin{abstract}
We discuss a linear-quadratic optimal control problem with pointwise control and state constraints. The state constraints are regularized by a Lavrentiev type regularization. The main results of the paper are estimates for the regularization error and the stability with respect to noisy data.
\end{abstract}

Keywords. Optimal control, linear-quadratic problems, state constraints, Lavrentiev regularization, perturbations, stability

Mathematics Subject Classification (2000). Primary 49M20, secondary 49K20, $49 \mathrm{~N} 10,90 \mathrm{C} 46$

\section{Introduction}

In this paper we consider the following elliptic control problem with pointwise state constraints and distributed control

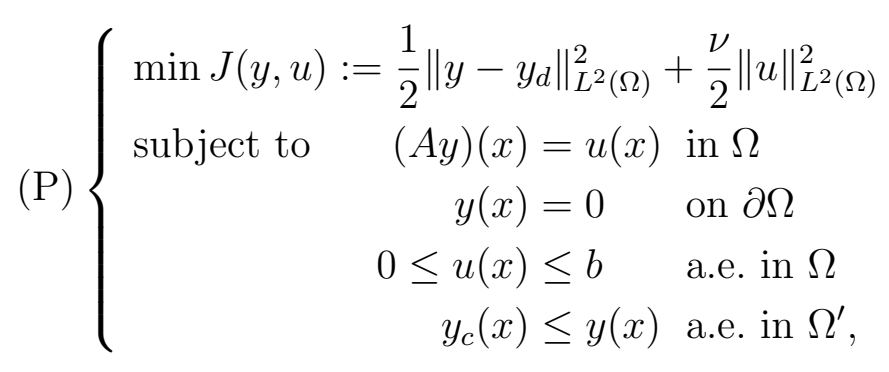

where $\Omega \subset \mathbb{R}^{N}, N \geq 2$, is a bounded domain with $C^{0,1}$ boundary $\partial \Omega$. We assume $\Omega^{\prime} \subset \Omega$ with $\operatorname{dist}\left(\Omega^{\prime}, \partial \Omega\right)>0$. This is motivated by the investigation of a homogeneous Dirchlet problem. Since the boundary data are fixed a bad choice of $y_{c}$ would be immediately lead to an empty feasible set. Conversely, a

A. Rösch: Universität Duisburg-Essen, Fachbereich Mathematik, Forsthausweg 2, D47057 Duisburg, Germany; arnd.roesch@uni-due.de

S. Cherednichenko: Universität Duisburg-Essen, Fachbereich Mathematik, Forsthausweg 2, D-47057 Duisburg, Germany; svetlana.cherednichenko@uni-due.de 
function $y_{c}$ which is bounded below by a positive number in a neighbourhood of the boundary $\partial \Omega$ would be equivalent to the problem under consideration, since the state $y$ is continuous.

The parameters $\nu$ and $b$ are fixed positive real numbers and $y_{d}$ is a fixed function from $L^{2}(\Omega)$. The state constraint $y_{c}$ is a function belonging to $L^{\infty}\left(\Omega^{\prime}\right)$ and $A$ is a uniformly elliptic differential operator. More precisely, it has the form

$$
A y(x)=-\sum_{i, j=1}^{n} D_{i}\left(a_{i j}(x) D_{j} y(x)\right)+c(x) y(x),
$$

where $D_{i}$ denotes the partial derivative with respect to $x_{i}$. Here $c$ is a given function in $L^{\infty}(\Omega)$ with $c(x) \geq 0$ a.e., and $a_{i j}$ belonging to $C^{0,1}(\bar{\Omega})$, and satisfying the conditions $a_{i j}=a_{j i}$ for all $i, j=1, \ldots, n$ with the ellipticity condition

$$
\sum_{i, j=1}^{n} a_{i j}(x) \xi_{i} \xi_{j} \geq \theta\|\xi\|^{2} \quad \forall(x, \xi) \in \Omega \times \mathbb{R}^{n},
$$

where $\theta$ is some positive constant.

The main difficulty in this problem is the pointwise state constraint $y(x) \geq$ $y_{c}(x)$. Theory and numerical treatment of such constraints are still a challenge. In this paper we use a Lavrentiev type regularization of the state constraints, introduced in Meyer, Rösch and Tröltzsch [6]. Let us remark that the Lavrentiev regularization is well studied for inverse problems, see Lavrentiev [4], Liu and Nashed [5], Janno [3], Tautenhahn [9], Nair and Tautenhahn [7].

For optimal control problems, the Lavrentiev type regularization is motivated by the following facts. Typically, mixed pointwise control-state constraints have better theoretical properties than state constrained problems. There are several situations known where the existence of measurable and bounded Lagrange multipliers can be shown, see Tröltzsch [10] and Rösch and Tröltzsch [8]. In contrast to this, Lagrange multipliers associated to pointwise state constraints can be expected only in measure spaces. Numerical tests show that the condition numbers of linear systems associated to the regularized problems are essentially smaller than such one for the unregularized problem. The high condition numbers for unregularized problems occur if the state constraint is active on an open subset. On such sets, the optimal control $\bar{u}$ is obtained by $A y_{c}=\bar{u}$, i.e., the data have to be differentiated twice. The Lavrentiev regularization of such an operator equation is studied in the theory of inverse problems and corresponding papers were already cited. We will see in this paper that the occurence of the pointwise bounds for the control $u$ stabilizes the problem. Therefore, a control of the regularization parameter with respect to the noice level is not essential. However, first numerical studies show that a reasonable balance between noice level, regularization parameter, and the discretization parameter improves the behavior of the involved numerical methods essentially. 
A family of optimal control problems with regularized state constraints is given by

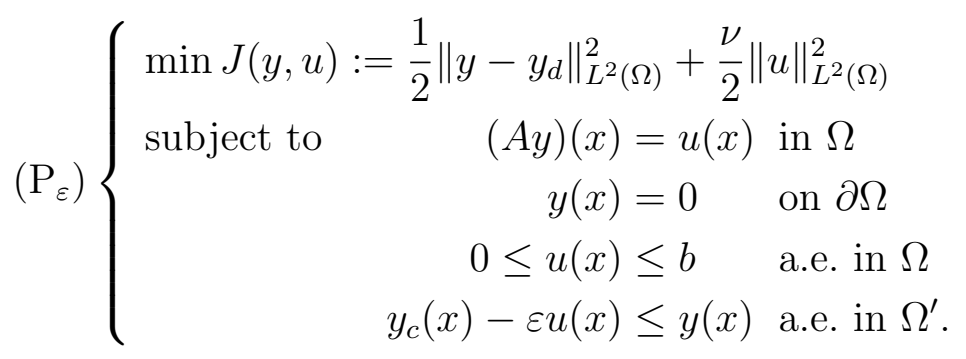

This family is characterized by a regularization parameter $\varepsilon>0$. Note that Problem $(\mathrm{P})$ is obtained for $\varepsilon=0$. In this paper we will derive estimates for the regularization error and prove stability properties. The used proving technique was introduced in a paper by Alt [1].

The paper is organized as follows. In Section 2 we state properties of the involved partial differential equations and the optimality conditions. Section 3 contains error estimates for the solutions of the regularized problems $\left(\mathrm{P}_{\varepsilon}\right)$ with respect to the solution of problem $(\mathrm{P})$. In Section 4, we will study the case of noisy data $y_{c}$ and $y_{d}$. Section 5 is devoted to feasible regularized solutions. The paper ends with numerical tests in Section 6.

\section{Optimality conditions}

We start with well known results for the weak formulation of the elliptic boundary value problem. A function $y \in V:=H_{0}^{1}(\Omega)$ is called a weak solution if

$$
a(y, v)=(u, v) \quad \forall v \in V
$$

is satisfied. Here $a: V \times V \rightarrow \mathbb{R}$ denotes the bilinear form defined by

$$
a(y, u)=\int_{\Omega} a_{i j}(x) D_{i} y(x) D_{j} v(x) d x+\int_{\Omega} c(x) y(x) v(x) d x .
$$

Lemma 2.1. Equation (1) has a unique solution $y \in V:=H_{0}^{1}(\Omega)$ for every $u \in L^{2}(\Omega)$. Moreover, the mapping $u \mapsto y$ is continuous from $L^{2}(\Omega)$ to $V$.

This statement follows immediately from the Lax-Milgram Theorem. It allows us to define a continuous control-to-state mapping $S: L^{2}(\Omega) \rightarrow V$ by $y=S u$ as a weak solution of (1).

Next, we introduce admissible control sets $U_{a d}$ for the Problem (P):

$$
U_{a d}:=\left\{u \in L^{2}(\Omega) \mid 0 \leq u(x) \leq b \text { a.e. in } \Omega,(S u)(x) \geq y_{c}(x) \text { a.e. in } \Omega^{\prime}\right\},
$$


and the admissible set $U_{a d}^{\varepsilon}$ for the modified problem $\left(\mathrm{P}_{\varepsilon}\right)$ :

$$
U_{a d}^{\varepsilon}:=\left\{u \in L^{2}(\Omega) \mid 0 \leq u(x) \leq b \text { a.e. in } \Omega, \varepsilon u+(S u)(x) \geq y_{c}(x) \text { a.e. in } \Omega^{\prime}\right\} .
$$

In general, these sets may be empty. To avoid this, we have to require the existence of at least one feasible point. However, for our analysis we need a slightly stronger assumption, the existence of a Slater point.

Assumption (A). There exists a control $\hat{u}(x)$ with $0 \leq \hat{u}(x) \leq b$ and $\hat{y}(x) \geq$ $y_{c}(x)+\tau$ a.e. in $\Omega^{\prime}, \tau>0$. Here $\hat{y}$ denotes the solution of (1) for the right-hand side $\hat{u}$.

Lemma 2.2. Assume that (A) holds. The optimal control problems (P) and $\left(\mathrm{P}_{\varepsilon}\right)$ admit uniquely determined solutions.

Proof. The objectives of both problems are strictly convex. Moreover, the admissible sets are closed, convex, and bounded in $L^{2}(\Omega)$. Consequently, the admissible sets are weakly compact in $L^{2}(\Omega)$. The objective is weakly lower semicontinuous. Therefore, the existence of optimal solutions is guaranteed if the admissible sets are non-empty. This is indeed the case. Because of

$$
\hat{y}(x)+\varepsilon \hat{u}(x) \geq \hat{y}(x) \geq y_{c}(x)+\tau \geq y_{c}(x),
$$

the pair $(\hat{y}, \hat{u})$ is admissible for both problems. The uniqueness of the solution is guaranteed by the strict convexity of the objectives.

To derive optimality conditions, we introduce an adjoint equation. All inequality constraints are contained in the set of admissible controls. Thanks to the non-standard definitions of the admissible sets, the adjoint equation of both problems is the same and contains no Lagrange multipliers. The adjoint equation is given in a weak formulation by

$$
a(v, p)=\left(y-y_{d}, v\right) \quad \forall v \in V
$$

or in a classical notation

$$
\begin{aligned}
\left(A^{*} p\right)(x) & =y(x)-y_{d}(x) & & \text { in } \Omega \\
p(x) & =0 & & \text { on } \partial \Omega .
\end{aligned}
$$

Here $A^{*}$ denotes the adjoint operator; since $a_{i j}=a_{j i}$ we have $A^{*}=A$.

Lemma 2.3. The adjoint equation (2) admits a unique weak solution $p \in V$.

In the following we will call the triple $(\bar{y}, \bar{u}, \bar{p})$ optimal solution of $(\mathrm{P})$. This means $\bar{u}$ is the optimal control and the corresponding state $\bar{y}$ and the corresponding adjoint state $\bar{p}$ are defined as solutions of (1) and (2). The optimal solution $\left(\bar{y}_{\varepsilon}, \bar{u}_{\varepsilon}, \bar{p}_{\varepsilon}\right)$ of $\left(\mathrm{P}_{\varepsilon}\right)$ is declared in exactly the same way. 
Lemma 2.4. A necessary and sufficient condition for the optimality of $(\bar{y}, \bar{u}, \bar{p})$ is given by

$$
(\bar{p}+\nu \bar{u}, u-\bar{u})_{L^{2}(\Omega)} \geq 0 \quad \text { for all } u \in U_{a d} .
$$

Analogously the optimality condition of $\left(y_{\varepsilon}, u_{\varepsilon}, p_{\varepsilon}\right)$ is given by

$$
\left(\bar{p}_{\varepsilon}+\nu \bar{u}_{\varepsilon}, u-\bar{u}_{\varepsilon}\right)_{L^{2}(\Omega)} \geq 0 \quad \text { for all } u \in U_{a d}^{\varepsilon} .
$$

Since this result is quite standard, we drop the proof.

Notation. If there is no risk of confusion, we denote in the following by $(.,)=$. $(., .)_{L^{2}(\Omega)}$ the inner product of $L^{2}(\Omega)$.

\section{Regularization error}

In this section we study the error of solutions of the regularized problems $\left(\mathrm{P}_{\varepsilon}\right)$ with respect to the solution of the original problem $(\mathrm{P})$.

Lemma 3.1. Assume that (A) is satisfied. Then for every $\varepsilon>0$, there exists a constant $\delta_{\varepsilon} \in(0,1)$ such that $u_{\delta}:=(1-\delta) \bar{u}_{\varepsilon}+\delta \hat{u}$ is feasible for $(\mathrm{P})$ for $\delta \in\left[\delta_{\varepsilon}, 1\right]$.

Proof. Since $\bar{u}_{\varepsilon}$ and $\hat{u}$ are feasible for $\left(\mathrm{P}_{\varepsilon}\right)$, the convex linear combination $u_{\delta}$ fulfills the control constraints $0 \leq u_{\delta} \leq b$. Consequently, we have only to check the state constraint. Here we know

$$
\varepsilon \bar{u}_{\varepsilon}+\bar{y}_{\varepsilon} \geq y_{c} \quad \Rightarrow \quad \bar{y}_{\varepsilon} \geq y_{c}-\varepsilon \bar{u}_{\varepsilon} .
$$

According to $(A)$, we have in addition $\hat{y} \geq y_{c}+\tau$. Due to the linearity of (1), we find $y_{\delta}=(1-\delta) \bar{y}_{\varepsilon}+\delta \hat{y}$, where $y_{\delta}$ denotes the solution of (1) with $u:=u_{\delta}$. This leads to

$$
\begin{aligned}
y_{\delta} & =(1-\delta) \bar{y}_{\varepsilon}+\delta \hat{y} \\
& \geq(1-\delta)\left(y_{c}-\varepsilon \bar{u}_{\varepsilon}\right)+\delta y_{c}+\delta \tau \\
& \geq y_{c}-\varepsilon \bar{u}_{\varepsilon}(1-\delta)+\delta \tau \\
& \geq y_{c}-\varepsilon(1-\delta) b+\delta \tau
\end{aligned}
$$

using $\left|u_{\varepsilon}\right| \leq b$ in the last line. Consequently, $u_{\delta}$ is feasible for $(\mathrm{P})$ if $\delta \tau-\varepsilon(1-\delta) b$ is positive. This takes place for $\delta \geq \frac{\varepsilon b}{\tau+\varepsilon b}$. Therefore, $\delta_{\varepsilon}$ can be defined as $\delta_{\varepsilon}:=$ $\frac{\varepsilon b}{\tau+\varepsilon b}$.

Lemma 3.2. For every $\varepsilon>0$ the solution $\bar{u}$ is feasible for $\left(\mathrm{P}_{\varepsilon}\right)$. 
Proof. We have only to check the mixed control-state constraint. Since $\bar{u}$ is feasible for $(\mathrm{P})$,

$$
\varepsilon \bar{u}+\bar{y} \geq \bar{y} \geq y_{c} \Rightarrow \varepsilon \bar{u}+\bar{y} \geq y_{c} \quad \text { a.e. in } \Omega
$$

is satisfied for every $\varepsilon>0$. Consequently, $\bar{u}$ is feasible for $\left(\mathrm{P}_{\varepsilon}\right)$ for arbitrary $\varepsilon>0$.

Next, we state the main result of this section.

Theorem 3.3. Assume that (A) is satisfied. Then, there exists a positive constant $C$ independent of $\varepsilon$ with

$$
\nu\left\|\bar{u}-\bar{u}_{\varepsilon}\right\|_{L^{2}(\Omega)}^{2}+\left\|\bar{y}-\bar{y}_{\varepsilon}\right\|_{L^{2}(\Omega)}^{2} \leq \varepsilon C .
$$

Proof. We start with the optimality condition (4). Due to Lemma 3.2, we can test this inequality with $\bar{u}$ :

$$
\left(\bar{p}_{\varepsilon}+\nu \bar{u}_{\varepsilon}, \bar{u}-\bar{u}_{\varepsilon}\right) \geq 0
$$

The construction of a test function for (3) is more difficult. Actually, we can not test (3) with $\bar{u}_{\varepsilon}$ since $\bar{u}_{\varepsilon}$ may be infeasible for $(\mathrm{P})$. However, from Lemma 3.1 we know the feasibility of $u_{\delta}$ for certain $\delta$. Inserting $u=u_{\delta}$ in (3), we find

$$
\left(\bar{p}+\nu \bar{u}, u_{\delta}-\bar{u}\right) \geq 0
$$

for $\delta \in\left[\delta_{\varepsilon}, 1\right]$. Adding (5) and (6), we find

$$
\left(\bar{p}_{\varepsilon}+\nu \bar{u}_{\varepsilon}, \bar{u}-\bar{u}_{\varepsilon}\right)+\left(\bar{p}+\nu \bar{u}, u_{\delta}-\bar{u}\right) \geq 0 .
$$

Next, we write this inequality in the form

$$
\left(\bar{p}_{\varepsilon}+\nu \bar{u}_{\varepsilon}, \bar{u}-\bar{u}_{\varepsilon}\right)+\left(\bar{p}+\nu \bar{u}, u_{\delta}-\bar{u}_{\varepsilon}\right)+\left(\bar{p}+\nu \bar{u}, \bar{u}_{\varepsilon}-\bar{u}\right) \geq 0
$$

or

$$
\left(\bar{p}-\bar{p}_{\varepsilon}, \bar{u}_{\varepsilon}-\bar{u}\right)+\left(\bar{p}+\nu \bar{u}, u_{\delta}-\bar{u}_{\varepsilon}\right)+\nu\left(\bar{u}-\bar{u}_{\varepsilon}, \bar{u}_{\varepsilon}-\bar{u}\right) \geq 0 .
$$

Consider the first term in (7). Since (1) holds true, we obtain $\left(\bar{p}-\bar{p}_{\varepsilon}, \bar{u}_{\varepsilon}-\bar{u}\right)=$ $a\left(\bar{p}-\bar{p}_{\varepsilon}, \bar{y}_{\varepsilon}-\bar{y}\right)$. On the other hand, (2) implies $a\left(\bar{p}-\bar{p}_{\varepsilon}, \bar{y}_{\varepsilon}-\bar{y}\right)=\left(\bar{y}-\bar{y}_{\varepsilon}, \bar{y}_{\varepsilon}-\bar{y}\right)$. Hence, we find $\left(\bar{p}-\bar{p}_{\varepsilon}, \bar{u}_{\varepsilon}-\bar{u}\right)=\left(\bar{y}-\bar{y}_{\varepsilon}, \bar{y}_{\varepsilon}-\bar{y}\right)=-\left\|\bar{y}-\bar{y}_{\varepsilon}\right\|_{L^{2}(\Omega)}^{2}$. Consequently, we can write $(7)$ in the form

$$
\nu\left\|\bar{u}-\bar{u}_{\varepsilon}\right\|_{L^{2}(\Omega)}^{2}+\left\|\bar{y}-\bar{y}_{\varepsilon}\right\|_{L^{2}(\Omega)}^{2} \leq\left(\nu \bar{u}+\bar{p}, u_{\delta}-\bar{u}_{\varepsilon}\right) .
$$

By the Cauchy-Schwarz inequality we arrive at

$$
\nu\left\|\bar{u}-\bar{u}_{\varepsilon}\right\|_{L^{2}(\Omega)}^{2}+\left\|\bar{y}-\bar{y}_{\varepsilon}\right\|_{L^{2}(\Omega)}^{2} \leq\|\nu \bar{u}+\bar{p}\|_{L^{2}(\Omega)}\left\|u_{\delta}-\bar{u}_{\varepsilon}\right\|_{L^{2}(\Omega)} .
$$


Moreover, we have

$$
\left\|u_{\delta}-\bar{u}_{\varepsilon}\right\|_{L^{2}(\Omega)}=\left\|(1-\delta) \bar{u}_{\varepsilon}+\delta \hat{u}-\bar{u}_{\varepsilon}\right\|_{L^{2}(\Omega)}=\left\|\delta\left(\hat{u}-\bar{u}_{\varepsilon}\right)\right\|_{L^{2}(\Omega)} \leq \delta b|\Omega|^{\frac{1}{2}} .
$$

For $\delta=\delta_{\varepsilon}=\frac{\varepsilon b}{\tau+\varepsilon b}$ we obtain

$$
\left\|u_{\delta}-\bar{u}_{\varepsilon}\right\|_{L^{2}(\Omega)} \leq|\Omega|^{\frac{1}{2}} \frac{\varepsilon b^{2}}{\tau+\varepsilon b} \leq|\Omega|^{\frac{1}{2}} \frac{\varepsilon b^{2}}{\tau} .
$$

Combining this inequality with (8), we get

$$
\nu\left\|\bar{u}-\bar{u}_{\varepsilon}\right\|_{L^{2}(\Omega)}^{2}+\left\|\bar{y}-\bar{y}_{\varepsilon}\right\|_{L^{2}(\Omega)}^{2} \leq \varepsilon C
$$

with $C=\frac{b^{2}}{\tau}|\Omega|^{\frac{1}{2}} \cdot\|\nu \bar{u}+\bar{p}\|_{L^{2}(\Omega)}$.

Remark 3.4. It is possible to find an a priori bound $\|\nu \bar{u}+\bar{p}\|_{L^{2}(\Omega)} \leq C^{\prime}$. We will work out this point in the next section.

\section{Stability of the solution with respect to perturbed data}

In this section we slightly change our original problem: We investigate perturbed data $y_{d}^{\sigma}$ and $y_{c}^{\sigma}$ instead of the original data $y_{d}$ and $y_{c}$. We are interested in error estimates separating the regularization error and the influence of the noice levels $\sigma_{c}$ and $\sigma_{d}$ :

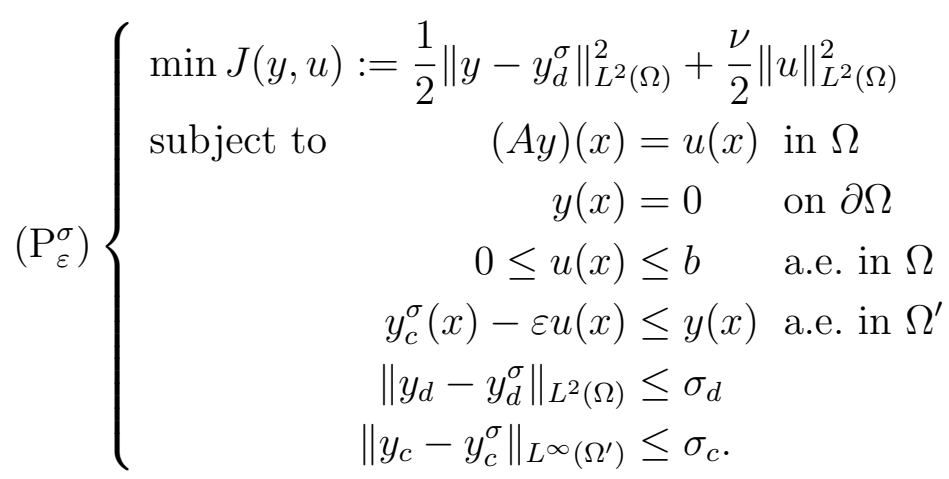

Now, Assumption (A) would be not strong enough to ensure the existence of a feasible control for $\left(\mathrm{P}_{\varepsilon}^{\sigma}\right)$. This difficulty occurs even for $\varepsilon=0$ because of perturbations in the data. An additional property of the Slater type point will overcome this problem. The new requirement is that the distance to the constraint $\tilde{\tau}$ is larger than the noice level $\sigma_{c}$ in the constraint.

Assumption (B). There exists a control $\tilde{u}(x)$ with $0 \leq \tilde{u}(x) \leq b, \tilde{y}(x) \geq$ $y_{c}^{\sigma}(x)+\tilde{\tau}$ a.e. in $\Omega^{\prime}$, and $\tau:=\tilde{\tau}-\sigma_{c}>0$. Here $\tilde{y}$ denotes the solution of (1) for the right-hand side $\tilde{u}$. 
Lemma 4.1. Let $\sigma_{c}, \sigma_{d}$, and $\varepsilon$ are fixed nonnegative numbers. Assume that (B) is valid. Then the problem $\left(\mathrm{P}_{\varepsilon}^{\sigma}\right)$ admits a uniquely determined solution.

Proof. As mentioned above, the main difficulty is to show the existence of a feasible point. However, Assumption (B) ensures that $\tilde{u}(x)$ is feasible for $\left(\mathrm{P}_{\varepsilon}^{\sigma}\right)$ :

$$
\tilde{y}(x) \geq y_{c}^{\sigma}(x)+\tilde{\tau} \geq y_{c}^{\sigma}(x) \geq y_{c}^{\sigma}(x)-\varepsilon \tilde{u}(x) .
$$

Moreover, $\tilde{u}(x)$ is also feasible for $(\mathrm{P})$ because of

$$
\tilde{y}(x) \geq y_{c}^{\sigma}(x)+\tilde{\tau} \geq y_{c}^{\sigma}(x)+\sigma_{c} \geq y_{c}(x) .
$$

The remaining part of the proof can be done along the lines of the proof of Lemma 2.2.

Next, we introduce the set of admissible controls $U_{a d}^{\varepsilon, \sigma}$ via $U_{a d}^{\varepsilon, \sigma}:=\left\{u \in L^{2}(\Omega) \mid 0 \leq u(x) \leq b\right.$ a.e. in $\Omega, \varepsilon u+(S u)(x) \geq y_{c}(x)$ a.e. in $\left.\Omega^{\prime}\right\}$.

Similar to Section 3, we will use the notation $\left(\bar{y}_{\varepsilon}^{\sigma}, \bar{u}_{\varepsilon}^{\sigma}, \bar{p}_{\varepsilon}^{\sigma}\right)$ for the optimal solution of $\left(\mathrm{P}_{\varepsilon}^{\sigma}\right)$.

Lemma 4.2. For the optimality of $\left(\bar{y}_{\varepsilon}^{\sigma}, \bar{u}_{\varepsilon}^{\sigma}, \bar{p}_{\varepsilon}^{\sigma}\right)$, a necessary and sufficient condition is given by

$$
\left(\bar{p}_{\varepsilon}^{\sigma}+\nu \bar{u}_{\varepsilon}^{\sigma}, u-\bar{u}_{\varepsilon}^{\sigma}\right) \geq 0 \quad \text { for all } u \in U_{a d}^{\varepsilon, \sigma} .
$$

This result can be obtained by standard arguments. Therefore, we drop the proof.

Lemma 4.3. Assume that (B) holds. Then for every $\varepsilon>0$, there exists a constant $\delta^{\sigma} \in(0,1)$ such that $u_{\delta}^{\sigma}:=(1-\delta) \bar{u}+\delta \tilde{u}$ is feasible for $\left(\mathrm{P}_{\varepsilon}^{\sigma}\right)$ for $\delta \in\left[\delta^{\sigma}, 1\right]$.

Proof. We check only the mixed control-state constraint. Let $y_{\delta}^{\sigma}$ be the solution of (1) for right-hand side $u_{\delta}^{\sigma}$. We start with

$$
\begin{aligned}
y_{\delta}^{\sigma} & =(1-\delta) \bar{y}+\delta \tilde{y} \\
& \geq(1-\delta) y_{c}+\delta\left(y_{c}^{\sigma}+\tilde{\tau}\right) \\
& \geq(1-\delta)\left(y_{c}^{\sigma}-\sigma_{c}\right)+\delta y_{c}^{\sigma}+\delta \tilde{\tau} \\
& \geq y_{c}^{\sigma}+\delta \tilde{\tau}-(1-\delta) \sigma_{c} .
\end{aligned}
$$

Consequently, $u_{\delta}^{\sigma}$ is feasible for $\left(\mathrm{P}_{\varepsilon}^{\sigma}\right)$ for $\delta \in\left[\delta^{\sigma}, 1\right]$ with $\delta^{\sigma}:=\frac{\sigma_{c}}{\sigma_{c}+\tilde{\tau}}$.

Lemma 4.4. Assume that (B) holds. Then for every $\varepsilon>0$, there exists a positive constant $\rho_{\varepsilon}^{\sigma}$ such that $u_{\rho}^{\sigma}:=(1-\rho) \bar{u}_{\varepsilon}^{\sigma}+\rho \tilde{u}$ is feasible for $(P)$ for every $\rho$ in $\left[\rho_{\varepsilon}^{\sigma}, 1\right]$. 
Proof. Again, we check only the mixed control-state constraint. Let $y_{\rho}^{\sigma}$ be the solution of (1) for right-hand side $u_{\rho}^{\sigma}$. Using (B), we find

$$
\begin{aligned}
y_{\rho}^{\sigma} & =(1-\rho) \bar{y}_{\varepsilon}^{\sigma}+\rho \tilde{y} \\
& \geq(1-\rho)\left(y_{c}^{\sigma}-\varepsilon \bar{u}_{\varepsilon}^{\sigma}\right)+\rho\left(y_{c}^{\sigma}+\tilde{\tau}\right) \\
& \geq y_{c}^{\sigma}-(1-\rho) \varepsilon \bar{u}_{\varepsilon}^{\sigma}+\rho \tilde{\tau} \\
& \geq y_{c}-\sigma_{c}-(1-\rho) \varepsilon b+\rho \tilde{\tau} \\
& \geq y_{c}-(1-\rho)\left(\varepsilon b+\sigma_{c}\right)+\rho \tau .
\end{aligned}
$$

Consequently, $u_{\rho}^{\sigma}$ is feasible for $(\mathrm{P})$ for $\rho \in\left[\rho_{\varepsilon}^{\sigma}, 1\right]$ with $\rho_{\varepsilon}^{\sigma}:=\frac{\varepsilon b+\sigma_{c}}{\varepsilon b+\sigma_{c}+\tau}$.

Theorem 4.5. Assume that Assumption (B) is satisfied. Then, there exist positive constants $C_{1}$ and $C_{2}$ independent of $\sigma_{d}$ and $\varepsilon$ with

$$
\nu\left\|\bar{u}-\bar{u}_{\varepsilon}^{\sigma}\right\|_{L^{2}(\Omega)}^{2}+\frac{1}{2}\left\|\bar{y}-\bar{y}_{\varepsilon}^{\sigma}\right\|_{L^{2}(\Omega)}^{2} \leq C_{1} \varepsilon+C_{2} \sigma_{c}+\frac{1}{2} \sigma_{d}^{2} .
$$

Proof. We start with the optimality condition (10). Due to Lemma 4.3, we can test this inequality with $u_{\delta}^{\sigma}$ for $\delta \in\left[\delta^{\sigma}, 1\right]$,

$$
\left(\bar{p}_{\varepsilon}^{\sigma}+\nu \bar{u}_{\varepsilon}^{\sigma}, u_{\delta}^{\sigma}-\bar{u}_{\varepsilon}^{\sigma}\right) \geq 0 .
$$

According to Lemma 4.4, we can test the optimality condition (3) with $u_{\rho}^{\sigma}$ for $\rho \in\left[\rho_{\varepsilon}^{\sigma}, 1\right]$,

$$
\left(\bar{p}+\nu \bar{u}, u_{\rho}^{\sigma}-\bar{u}\right) \geq 0 .
$$

Adding (11) and (12), we find

$$
\left(\bar{p}_{\varepsilon}^{\sigma}+\nu \bar{u}_{\varepsilon}^{\sigma}, u_{\delta}^{\sigma}-\bar{u}_{\varepsilon}^{\sigma}\right)+\left(\bar{p}+\nu \bar{u}, u_{\rho}^{\sigma}-\bar{u}\right) \geq 0 .
$$

Next we rewrite this inequality as

$\left(\bar{p}_{\varepsilon}^{\sigma}+\nu \bar{u}_{\varepsilon}^{\sigma}, u_{\delta}^{\sigma}-\bar{u}\right)+\left(\bar{p}_{\varepsilon}^{\sigma}+\nu \bar{u}_{\varepsilon}^{\sigma}, \bar{u}-\bar{u}_{\varepsilon}^{\sigma}\right)+\left(\bar{p}+\nu \bar{u}, u_{\rho}^{\sigma}-\bar{u}_{\varepsilon}^{\sigma}\right)+\left(\bar{p}+\nu \bar{u}, \bar{u}_{\varepsilon}^{\sigma}-\bar{u}\right) \geq 0$.

Combining the second and the last term, the inequality can be written in the form

$$
\left(\bar{p}_{\varepsilon}^{\sigma}+\nu \bar{u}_{\varepsilon}^{\sigma}, u_{\delta}^{\sigma}-\bar{u}\right)+\left(\bar{p}+\nu \bar{u}, u_{\rho}^{\sigma}-\bar{u}_{\varepsilon}^{\sigma}\right)+\nu\left(\bar{u}-\bar{u}_{\varepsilon}^{\sigma}, \bar{u}_{\varepsilon}^{\sigma}-\bar{u}\right)+\left(\bar{p}-\bar{p}_{\varepsilon}^{\sigma}, \bar{u}_{\varepsilon}^{\sigma}-\bar{u}\right) \geq 0 .
$$

We start with the estimation of last term in (13). Since (1) holds true, we have

$$
\left(\bar{p}-\bar{p}_{\varepsilon}^{\sigma}, \bar{u}_{\varepsilon}^{\sigma}-\bar{u}\right)=a\left(\bar{y}_{\varepsilon}^{\sigma}-\bar{y}, \bar{p}-\bar{p}_{\varepsilon}^{\sigma}\right) .
$$

On the other hand, the adjoint equation (2) implies

$$
a\left(\bar{y}_{\varepsilon}^{\sigma}-\bar{y}, \bar{p}-\bar{p}_{\varepsilon}^{\sigma}\right)=\left(\bar{y}-\bar{y}_{\varepsilon}^{\sigma}, \bar{y}_{\varepsilon}^{\sigma}-\bar{y}\right)+\left(y_{d}^{\sigma}-y_{d}, \bar{y}_{\varepsilon}^{\sigma}-\bar{y}\right) .
$$


From (14) and (15), we conclude

$$
\left(\bar{p}-\bar{p}_{\varepsilon}^{\sigma}, u_{\varepsilon}^{\sigma}-\bar{u}\right)=-\left\|\bar{y}_{\varepsilon}^{\sigma}-\bar{y}\right\|_{L^{2}(\Omega)}^{2}+\left(y_{d}-y_{d}^{\sigma}, \bar{y}-\bar{y}_{\varepsilon}^{\sigma}\right) .
$$

Consequently, we can write (13) in the form

$$
\begin{aligned}
& \nu\left\|\bar{u}-\bar{u}_{\varepsilon}^{\sigma}\right\|_{L^{2}(\Omega)}^{2}+\left\|\bar{y}-\bar{y}_{\varepsilon}^{\sigma}\right\|_{L^{2}(\Omega)}^{2} \\
& \quad \leq\left(\nu \bar{u}+\bar{p}, u_{\rho}^{\sigma}-\bar{u}_{\varepsilon}^{\sigma}\right)+\left(\bar{p}_{\varepsilon}^{\sigma}+\nu \bar{u}_{\varepsilon}^{\sigma}, u_{\delta}^{\sigma}-\bar{u}\right)+\left(y_{d}-y_{d}^{\sigma}, \bar{y}-\bar{y}_{\varepsilon}^{\sigma}\right) .
\end{aligned}
$$

Next, we estimate the three inner products using the special choice $\rho=\rho_{\varepsilon}^{\sigma}$. We start with

$$
\begin{aligned}
\left(\nu \bar{u}+\bar{p}, u_{\rho}^{\sigma}-\bar{u}_{\varepsilon}^{\sigma}\right) & \leq\|\nu \bar{u}+\bar{p}\|_{L^{2}(\Omega)}\left\|u_{\rho}^{\sigma}-\bar{u}_{\varepsilon}^{\sigma}\right\|_{L^{2}(\Omega)} \\
& =\rho\|\nu \bar{u}+\bar{p}\|_{L^{2}(\Omega)}\left\|\tilde{u}-\bar{u}_{\varepsilon}^{\sigma}\right\|_{L^{2}(\Omega)} \\
& \leq \frac{\varepsilon b+\sigma_{c}}{\varepsilon b+\sigma_{c}+\tau} b|\Omega|^{\frac{1}{2}}\|\nu \bar{u}+\bar{p}\|_{L^{2}(\Omega)} \\
& \leq \frac{\varepsilon b+\sigma_{c}}{\tau} b|\Omega|^{\frac{1}{2}}\|\nu \bar{u}+\bar{p}\|_{L^{2}(\Omega)}
\end{aligned}
$$

Estimating the second term, we find

$$
\begin{aligned}
\left(\bar{p}_{\varepsilon}^{\sigma}+\nu \bar{u}_{\varepsilon}^{\sigma}, u_{\delta}^{\sigma}-\bar{u}\right) & \leq\left\|\bar{p}_{\varepsilon}^{\sigma}+\nu \bar{u}_{\varepsilon}^{\sigma}\right\|_{L^{2}(\Omega)}\left\|u_{\delta}^{\sigma}-\bar{u}\right\|_{L^{2}(\Omega)} \\
& \leq \delta\left\|\bar{p}_{\varepsilon}^{\sigma}+\nu \bar{u}_{\varepsilon}^{\sigma}\right\|_{L^{2}(\Omega)}\|\tilde{u}-\bar{u}\|_{L^{2}(\Omega)} \\
& \leq \frac{\sigma_{c}}{\sigma_{c}+\tilde{\tau}} b|\Omega|^{\frac{1}{2}}\left\|\bar{p}_{\varepsilon}^{\sigma}+\nu \bar{u}_{\varepsilon}^{\sigma}\right\|_{L^{2}(\Omega)} \\
& \leq \frac{\sigma_{c}}{\tilde{\tau}} b|\Omega|^{\frac{1}{2}}\left\|\bar{p}_{\varepsilon}^{\sigma}+\nu \bar{u}_{\varepsilon}^{\sigma}\right\|_{L^{2}(\Omega)}
\end{aligned}
$$

for the setting $\delta=\delta^{\sigma}$. The remaining third term will be estimated by Young's inequality

$$
\left(y_{d}-y_{d}^{\sigma}, \bar{y}-\bar{y}_{\varepsilon}^{\sigma}\right) \leq \frac{1}{2} \sigma_{d}^{2}+\frac{1}{2}\left\|\bar{y}-\bar{y}_{\varepsilon}^{\sigma}\right\|_{L^{2}(\Omega)}^{2} .
$$

Inserting (18),(19), and (20) in (17), we end up with

$$
\nu\left\|\bar{u}-\bar{u}_{\varepsilon}^{\sigma}\right\|_{L^{2}(\Omega)}^{2}+\frac{1}{2}\left\|\bar{y}_{\varepsilon}^{\sigma}-\bar{y}\right\|_{L^{2}(\Omega)}^{2} \leq C_{1} \varepsilon+C_{2} \sigma_{c}+\frac{1}{2} \sigma_{d}^{2}
$$

with $C_{1}=\frac{b^{2}}{\tau}|\Omega|^{\frac{1}{2}} \cdot\|\nu \bar{u}+\bar{p}\|_{L^{2}(\Omega)}, C_{2}=\frac{b}{\tau}|\Omega|^{\frac{1}{2}} \cdot\|\nu \bar{u}+\bar{p}\|_{L^{2}(\Omega)}+\frac{b}{\tilde{\tau}}|\Omega|^{\frac{1}{2}} \cdot\left\|\bar{p}_{\varepsilon}^{\sigma}+\nu \bar{u}_{\varepsilon}^{\sigma}\right\|_{L^{2}(\Omega)}$. This completes the proof.

Remark 4.6. Note that the constants $C_{1}$ and $C_{2}$ depend on $\sigma_{c}$ via $\tau$. However, if we fix a certain $\tilde{\sigma}_{c}:=\sigma_{c}$ and a corresponding $\tau$, then the statement of Theorem 4.5 becomes independent of $\sigma_{c}$ for $\sigma_{c} \in\left[0, \tilde{\sigma}_{c}\right]$. 
Corollary 4.7. Assume that (B) is satisfied. Then there exists a uniform bound $K$ such that

$$
\begin{aligned}
\|\nu \bar{u}+\bar{p}\| & \leq K \\
\left\|\nu u_{\varepsilon}^{\sigma}+p_{\varepsilon}^{\sigma}\right\| & \leq K .
\end{aligned}
$$

Proof. Since $\tilde{u}$ is feasible for $(\mathrm{P})$ and $\left(\mathrm{P}_{\varepsilon}^{\sigma}\right)$, we know

$$
\begin{aligned}
J(\bar{y}, \bar{u}) & \leq J(\tilde{y}, \tilde{u})=: J \\
J\left(y_{\varepsilon}^{\sigma}, u_{\varepsilon}^{\sigma}\right) & \leq J(\tilde{y}, \tilde{u})=J .
\end{aligned}
$$

The first inequality means $\frac{1}{2}\left\|\bar{y}-y_{d}\right\|_{L^{2}(\Omega)}^{2}+\frac{\nu}{2}\|\bar{u}\|_{L^{2}(\Omega)}^{2} \leq J$ and implies

$$
\begin{aligned}
\left\|\bar{y}-y_{d}\right\|_{L^{2}(\Omega)} & \leq \sqrt{2 J} \\
\|\bar{u}\|_{L^{2}(\Omega)} & \leq \sqrt{\frac{2 J}{\nu}} .
\end{aligned}
$$

Using $(21)$, we obtain $\|\bar{p}\|_{L^{2}(\Omega)} \leq \bar{C}\left\|\bar{y}-y_{d}\right\|_{L^{2}(\Omega)} \leq \bar{C} \sqrt{2 J}$. Finally we arrive at

$$
\|\nu \bar{u}+\bar{p}\| \leq \nu\|\bar{u}\|+\|\bar{p}\| \leq \sqrt{2 \nu J}+\bar{C} \sqrt{2 J} .
$$

Proceeding as above for $\left\|\nu u_{\varepsilon}^{\sigma}+p_{\varepsilon}^{\sigma}\right\|_{L^{2}(\Omega)}$ we receive the inequality

$$
\left\|\nu u_{\varepsilon}^{\sigma}+p_{\varepsilon}^{\sigma}\right\|_{L^{2}(\Omega)} \leq \sqrt{2 \nu J}+\bar{C} \sqrt{2 J} .
$$

Hense, we can choose $K:=\sqrt{2 \nu J}+\bar{C} \sqrt{2 J}$.

\section{Feasible regularized solutions}

Until now, the solutions of the introduced regularized problems are in general not feasible for $(\mathrm{P})$. In this section, we will discuss two ways to construct feasible solutions. The first way is motivated by the proving technique of the last sections. Here, we constructed feasible solutions for $(\mathrm{P})$ based on the solutions of the regularized problems. We start with the solution of the problem $\left(\mathrm{P}_{\varepsilon}\right)$.

Lemma 5.1. The following estimate for the regularization is valid:

$$
\nu\left\|\bar{u}-u_{\delta}\right\|_{L^{2}(\Omega)}^{2}+\left\|\bar{y}-y_{\delta}\right\|_{L^{2}(\Omega)}^{2} \leq c \varepsilon
$$

provided that the parameter $\delta$ is chosen as $\delta=\delta_{\varepsilon}=\frac{\varepsilon b^{2}}{\tau+\varepsilon b}$. Here, $u_{\delta}$ is the function introduced in Lemma 3.1. 
Proof. The desired property is obtained by Theorem 3.3 and the triangle inequality. Moreover, we conclude from (9)

$$
\left\|u_{\delta}-\bar{u}_{\varepsilon}\right\|_{L^{2}(\Omega)} \leq|\Omega|^{\frac{1}{2}} \frac{\varepsilon b^{2}}{\tau+\varepsilon b} \leq c \varepsilon .
$$

A similar estimate is valid for the state.

Next, we present a similar result for $\left(\mathrm{P}_{\varepsilon}^{\sigma}\right)$

Lemma 5.2. The following estimate for the regularization is valid:

$$
\nu\left\|\bar{u}-u_{\rho}^{\sigma}\right\|_{L^{2}(\Omega)}^{2}+\left\|\bar{y}-y_{\rho}^{\sigma}\right\|_{L^{2}(\Omega)}^{2} \leq c_{1} \varepsilon+c_{2} \sigma_{c}+\frac{1}{2} \sigma_{d}^{2}
$$

provided that the parameters $\rho$ is chosen as $\rho=\rho_{\varepsilon}^{\sigma}=\frac{\varepsilon b+\sigma_{c}}{\varepsilon b+\sigma_{c}+\tau}$. Here, $u_{\delta}$ is the function introduced in Lemma 3.1.

This lemma can be shown with similar arguments like for Lemma 5.1. Let us remark that an alternative error estimation technique is available for the regularized Problem $\left(\mathrm{P}_{\varepsilon}\right)$ :

Corollary 5.3. Since $\left(y_{\delta}, u_{\delta}\right)$ is feasible for $(\mathrm{P})$ and $(\bar{y}, \bar{u})$ is feasible for $\left(\mathrm{P}_{\varepsilon}\right)$ for every $\varepsilon>0$ the following inequality is satisfied:

$$
J\left(\bar{y}_{\varepsilon}, \bar{u}_{\varepsilon}\right) \leq J(\bar{y}, \bar{u}) \leq J\left(y_{\delta}, u_{\delta}\right) .
$$

This relation can also be used to obtain the results of Theorem 3.3 and Lemma 5.1.

Let us discuss a second approach to construct feasible regularized solutions for $(\mathrm{P})$. We replace $\left(\mathrm{P}_{\varepsilon}\right)$ by $\left(\mathrm{P}_{\varepsilon}^{\prime}\right)$ :

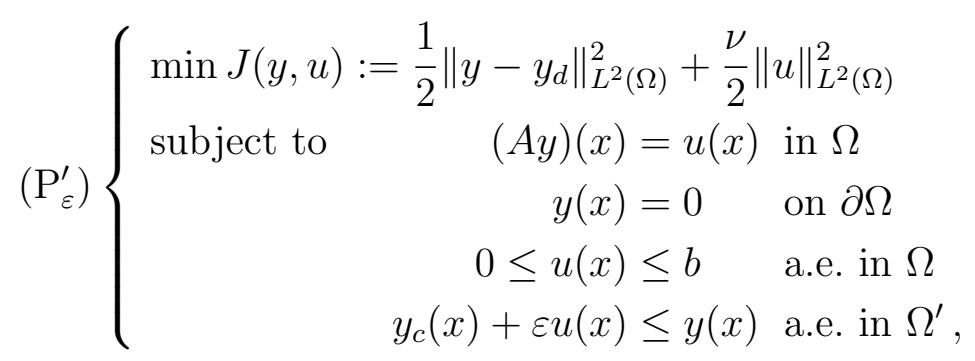

where only the sign in the mixed constrained has changed.

Now the situation with respect to the feasibility changes: Feasible controls for $\left(\mathrm{P}_{\varepsilon}^{\prime}\right)$ are automatically feasible for $(\mathrm{P})$. A drawback of this approach is that the existence of a feasible point for $\left(\mathrm{P}_{\varepsilon}^{\prime}\right)$ can only be guaranteed for sufficiently small $\varepsilon$. More precisely, if Assumption (A) is satisfied, then the existence of a feasible control can be shown for $\varepsilon \leq \frac{\tau}{\|\hat{u}\|_{L}^{\infty}(\Omega)}$. 
Theorem 5.4. Assume that (A) is satisfied. Then the estimate

$$
\nu\left\|\bar{u}-\bar{u}_{\varepsilon}\right\|_{L^{2}(\Omega)}^{2}+\left\|\bar{y}-\bar{y}_{\varepsilon}\right\|_{L^{2}(\Omega)}^{2} \leq \varepsilon C
$$

is valid with a positive constant $C$ independent of $\varepsilon$ for $\varepsilon \leq \frac{\tau}{\|\hat{u}\|_{L^{\infty}(\Omega)}}$.

The result can be obtained along the lines of Theorem 3.3. We have to change the problem once more if we want to deal with noisy data:

$$
\left(\mathrm{P}_{\varepsilon}^{\sigma^{\prime}}\right)\left\{\begin{aligned}
\min J(y, u):=\frac{1}{2}\left\|y-y_{d}^{\sigma}\right\|_{L^{2}(\Omega)}^{2}+ & \frac{\nu}{2}\|u\|_{L^{2}(\Omega)}^{2} \\
(A y)(x) & =u(x) \text { in } \Omega \\
y(x) & =0 \quad \text { on } \partial \Omega \\
\text { subject to } & \leq u(x) \leq b \quad \text { a.e. in } \Omega \\
y_{c}^{\sigma}(x)+\varepsilon u(x)+\sigma_{c} & \leq y(x) \text { a.e. in } \Omega^{\prime} \\
\left\|y_{d}-y_{d}^{\sigma}\right\|_{L^{2}(\Omega)} & \leq \sigma_{d} \\
\left\|y_{c}-y_{c}^{\sigma}\right\|_{L^{\infty}\left(\Omega^{\prime}\right)} & \leq \sigma_{c} .
\end{aligned}\right.
$$

The additional addend $\sigma_{c}$ ensures the feasibility of the solution of $\left(\mathrm{P}_{\varepsilon}^{\sigma^{\prime}}\right)$ for $(\mathrm{P})$. The existence of feasible solution for $\left(\mathrm{P}_{\varepsilon}^{\sigma^{\prime}}\right)$ can be ensured if Assumption (B) is satisfied and if $\varepsilon$ is sufficiently small, i.e., $\varepsilon \leq \frac{\tau}{\|\tilde{u}\|_{L^{\infty}(\Omega)}}$ with the quantity $\tau$ defined in Assumption (B).

Theorem 5.5. Assume that (B) is satisfied. Then the estimate

$$
\nu\left\|\bar{u}-\bar{u}_{\varepsilon}^{\sigma^{\prime}}\right\|_{L^{2}(\Omega)}^{2}+\left\|\bar{y}-\bar{y}_{\varepsilon}^{\sigma \prime}\right\|_{L^{2}(\Omega)}^{2} \leq c_{1} \varepsilon+c_{2} \sigma_{c}+\frac{1}{2} \sigma_{d}^{2}
$$

is valid for $\varepsilon \leq \frac{\tau}{\|\tilde{u}\|_{L^{\infty}(\Omega)}}$.

This result can be obtained along the lines of the proof of Theorem 4.5.

\section{Numerical tests}

We slightly modify the problem for the numerical tests:

$$
J(y, u)=\frac{1}{2}\left\|y-y_{d}\right\|_{L^{2}(\Omega)}^{2}+\frac{\nu}{2}\left\|u-u_{d}\right\|_{L^{2}(\Omega)}^{2}
$$

and

$$
\begin{aligned}
(-\Delta y)(x) & =u(x)+f(x) & & \text { in } \Omega, \\
y(x) & =0 & & \text { on } \partial \Omega,
\end{aligned}
$$

that means we introduce functions $f$ and $u_{d}$. This allows us to construct an example where the exact solution is known for the unregularized problem. 
For this purpose we need a different formulation of the optimality system. This formulation was introduced by Casas [2] and contains a Borel measure $\mu$ as additional source term of an adjoint partial differential equation:

$$
\begin{aligned}
-\Delta \hat{p} & =\bar{y}-\bar{y}_{d}-\mu & & \text { in } \Omega \\
\hat{p} & =0 & & \text { on } \partial \Omega .
\end{aligned}
$$

The optimality condition reads now

$$
\bar{u}(x)=\Pi_{[a, b]}\left(-\frac{1}{\nu} \hat{p}(x)+u_{d}\right)
$$

where the pointwise projection $\Pi_{[a, b]}$ is defined by

$$
\Pi_{[a, b]} f(x):=\max (a, \min (b, f(x))) .
$$

Moreover the following condition for the multiplier have to be fullfilled

$$
\int_{\Omega^{\prime}}\left(y-y_{c}\right) d \mu=0, \quad \mu \geq 0 .
$$

The following example is constructed in such a way that these necessary and sufficient optimality conditions are satisfied. Next, we present a complete list of functions appearing in our example. It is easy to show that these data satisfy the optimality system.

The optimal state is given by

$$
\bar{y}(x)=\sin \pi x_{1} \sin \pi x_{2} .
$$

The example depends on a parameter $c \in(0,1)$. In our tests we always set $c=0.6$. The function $y_{c}$ is given by

$$
y_{c}(x)= \begin{cases}\bar{y}(x) & \text { if } \quad \bar{y}(x) \geq c \\ 2 \bar{y}(x)-c & \text { if } \quad \bar{y}(x)<c .\end{cases}
$$

The desired state $y_{d}$ is a discontinuous function

$$
y_{d}(x)= \begin{cases}\bar{y}(x)-1 & \text { if } \quad \bar{y}(x) \geq c \\ \left(4 \nu \pi^{4}+1\right) \bar{y}(x) & \text { if } \quad \bar{y}(x)<c .\end{cases}
$$

The adjoint state $\hat{p}$ is a function with a kink:

$$
\hat{p}(x)= \begin{cases}-2 \pi^{2} \nu c & \text { if } \quad \bar{y}(x) \geq c \\ -2 \pi^{2} \nu \bar{y}(x) & \text { if } \quad \bar{y}(x)<c .\end{cases}
$$


Consequently, the Lagrange multiplier contains a line measure concentrated on the curve $\bar{y}(x)=c$. The function part of the Lagrange multiplier is given by

$$
\mu(x)=\left\{\begin{array}{lll}
1 & \text { if } \quad \bar{y}(x) \geq c \\
0 & \text { if } & \bar{y}(x)<c .
\end{array}\right.
$$

For convenience, we define a function $v$ by

$$
v(x)=2 \pi^{2} \sin \pi x_{1} \sin \pi x_{2}-\kappa
$$

with a positive $\kappa$ (in the computations we used $\kappa=5$ ). Next we set

$$
f(x)=\left\{\begin{array}{lll}
v+\kappa & \text { if } \quad v(x) \leq 0 \\
\kappa & \text { if } \quad v(x) \in(0, b) \\
v+\kappa-b & \text { if } \quad v(x) \geq b
\end{array}\right.
$$

and

$$
\bar{u}(x)=\left\{\begin{array}{lll}
0 & \text { if } & v(x) \leq 0 \\
v & \text { if } & v(x) \in(0, b) \\
b & \text { if } & v(x) \geq b .
\end{array}\right.
$$

Moreover, we have the function

$$
u_{d}(x)=\left\{\begin{array}{lll}
-2 \pi^{2} c+v(x) & \text { if } & \bar{y}(x) \geq c \\
-\kappa & \text { if } & \bar{y}(x)<c .
\end{array}\right.
$$

The constant $b=100$ is chosen in a way that the upper bound is not active.
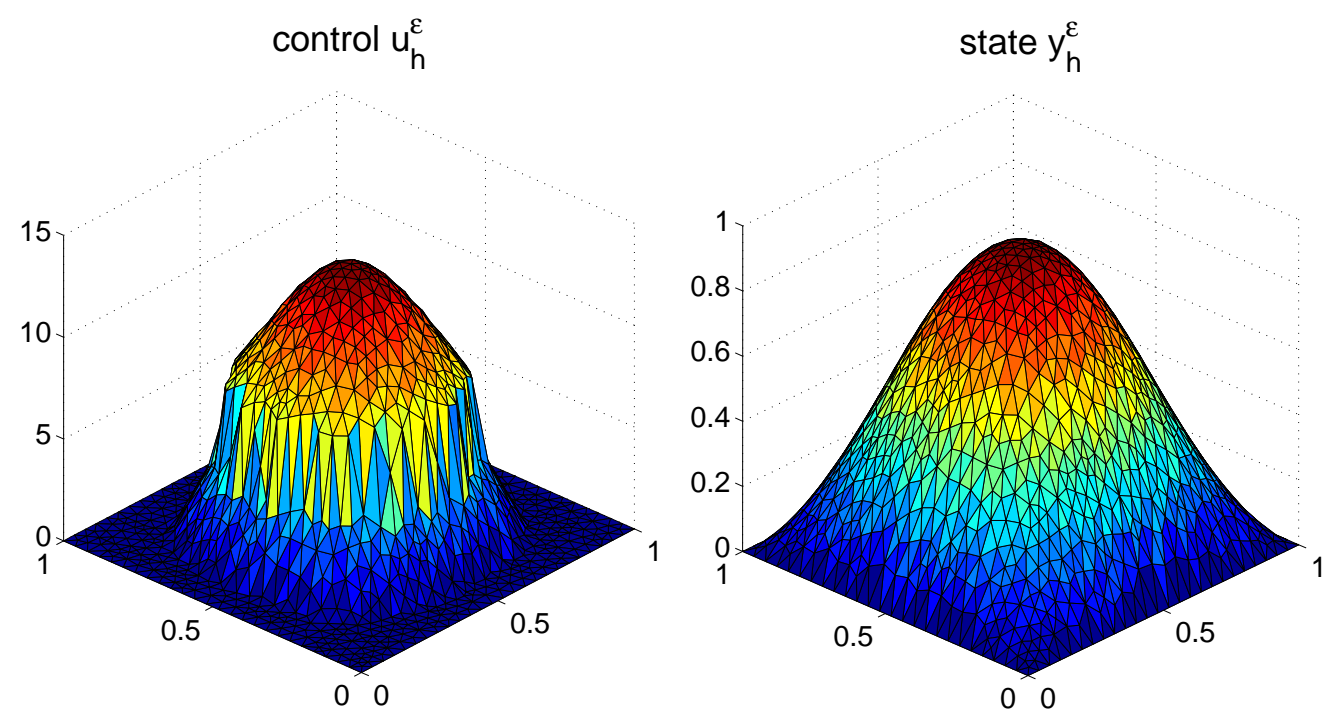

Figure 1: Optimal numerical solution for $\varepsilon=0.005$ 
We used a uniform finite element mesh with triangles. Moreover, we discretized the control, state and adjoint state by piecewise linear functions. The mesh size was $h=0.04$. Furthermore, we used $\nu=0.1$. The regularization parameter $\varepsilon$ varies in an interval where the regularization error is larger than the discretization error. Figure 1 shows the computed optimal control and the optimal state for $\varepsilon=0.005$. The computed error behavior is presented in Figure 2 and Table 1.
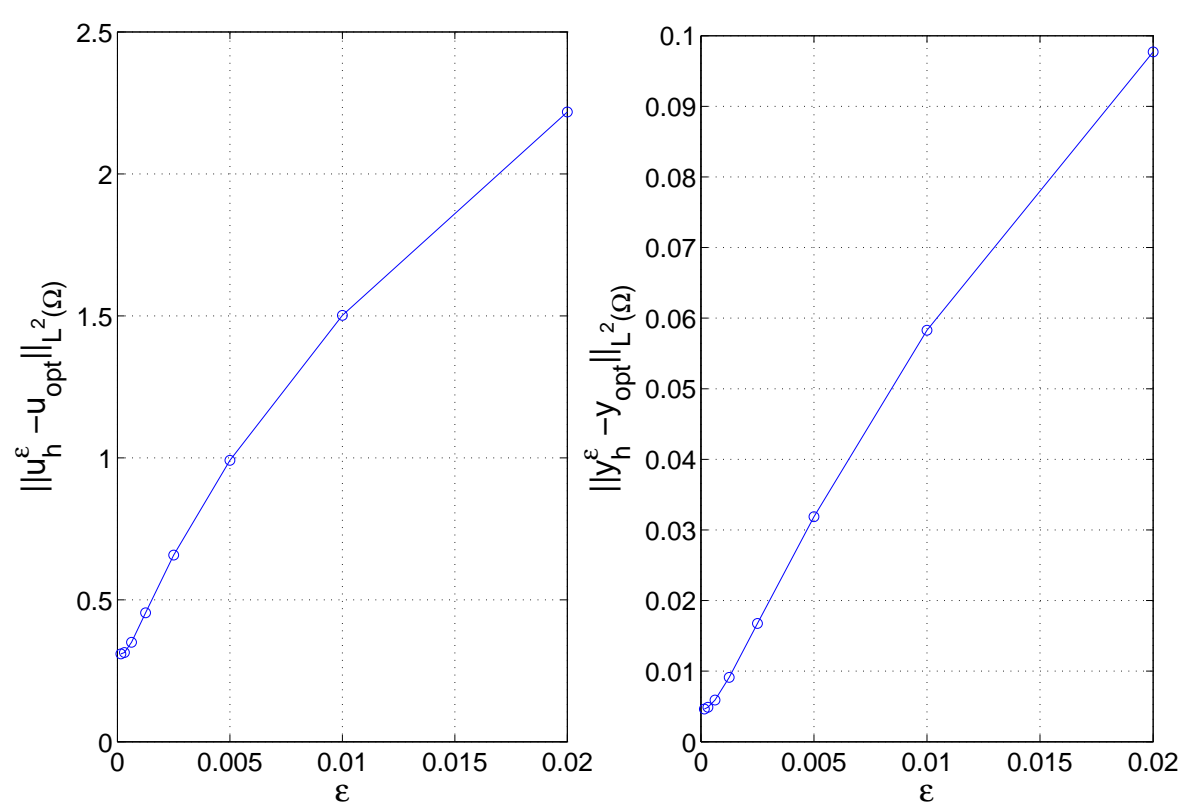

Figure 2: Error behaviour with respect to $\varepsilon$

\begin{tabular}{|c|c|c|c|c|}
\hline$\varepsilon$ & $\left\|\bar{u}-u_{h}^{\varepsilon}\right\|$ & $\frac{\left\|\bar{u}-u_{h}^{\varepsilon}\right\|}{\sqrt{\varepsilon}}$ & $\left\|\bar{y}-y_{h}^{\varepsilon}\right\|$ & $\frac{\left\|\bar{y}-y_{h}^{\varepsilon}\right\|}{\sqrt{\varepsilon}}$ \\
\hline \hline $2^{1} \cdot 10^{-2}$ & $2.2180 e+0$ & 15.684 & $9.7706 e-2$ & 0.69089 \\
\hline $2^{0} \cdot 10^{-2}$ & $1.5018 e+0$ & 15.018 & $5.8289 e-2$ & 0.58289 \\
\hline $2^{-1} \cdot 10^{-2}$ & $9.9099 e-1$ & 14.015 & $3.1875 e-2$ & 0.45079 \\
\hline $2^{-2} \cdot 10^{-2}$ & $6.5758 e-1$ & 13.152 & $1.6745 e-2$ & 0.33490 \\
\hline $2^{-3} \cdot 10^{-2}$ & $4.5411 e-1$ & 12.844 & $9.1220 e-3$ & 0.25801 \\
\hline $2^{-4} \cdot 10^{-2}$ & $3.5025 e-1$ & 14.010 & $5.9091 e-3$ & 0.23636 \\
\hline $2^{-5} \cdot 10^{-2}$ & $3.1421 e-1$ & 17.774 & $4.8770 e-3$ & 0.27588 \\
\hline
\end{tabular}

Table 1: $\varepsilon$-dependency $\left(\|\cdot\|\right.$ means $\left.\|\cdot\|_{L^{2}(\Omega)}\right)$ 
Next, we perturb the function $y_{c}$. We add some random pertubations to $y_{c}$ such that $\left\|y_{c}^{\sigma}-y_{c}\right\|_{L^{\infty}(\Omega)} \leq \sigma_{c}$ is valid. We set the regularization parameter $\varepsilon=2^{-8} \cdot 10^{-2}$ in order to decrease the regularization error generated by $\varepsilon$. Figure 3 and Table 2 illustrate the expected behavior of the numerical solutions with respect to $\sigma_{c}$.
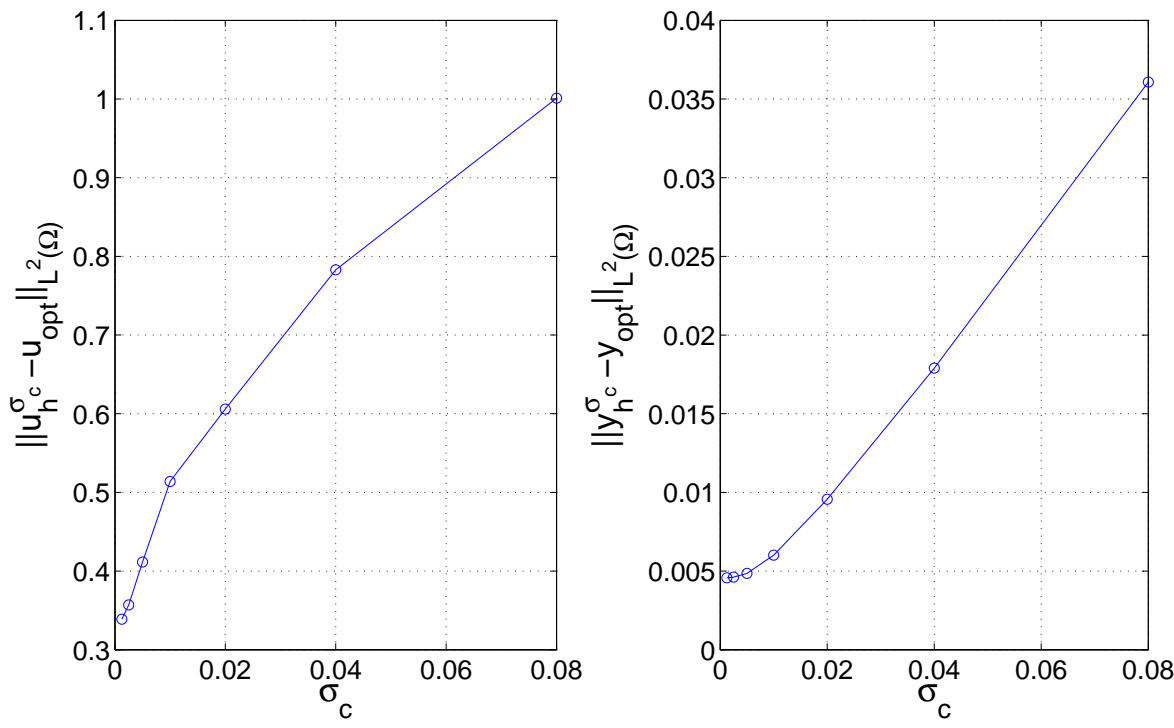

Figure 3: Error behavior with respect to $\sigma_{c}$

\begin{tabular}{|c|c|c|c|c|}
\hline$\sigma_{c}$ & $\left\|\bar{u}-u_{h}^{\varepsilon, \sigma_{c}}\right\|$ & $\frac{\left\|\bar{u}-u_{h}^{\varepsilon, \sigma_{c}}\right\|}{\sqrt{\sigma_{c}}}$ & $\left\|\bar{y}-y_{h}^{\varepsilon, \sigma_{c}}\right\|$ & $\frac{\left\|\bar{y}-y_{h}^{\varepsilon, \sigma_{c}}\right\|}{\sqrt{\sigma_{c}}}$ \\
\hline \hline $2^{3} \cdot 10^{-2}$ & $1.0011 e+0$ & 3.5394 & $3.6082 e-2$ & 0.1276 \\
\hline $2^{2} \cdot 10^{-2}$ & $7.8281 e-1$ & 3.9140 & $1.7899 e-2$ & 0.0895 \\
\hline $2^{1} \cdot 10^{-2}$ & $6.0590 e-1$ & 4.2844 & $9.5663 e-3$ & 0.0676 \\
\hline $2^{0} \cdot 10^{-2}$ & $5.1377 e-1$ & 5.1377 & $6.0098 e-3$ & 0.0601 \\
\hline $2^{-1} \cdot 10^{-2}$ & $4.1156 e-1$ & 5.8204 & $4.8575 e-3$ & 0.0687 \\
\hline $2^{-2} \cdot 10^{-2}$ & $3.5701 e-1$ & 7.1401 & $4.6160 e-3$ & 0.0923 \\
\hline
\end{tabular}

Table 2: $\sigma_{c^{-}}$dependency $\left(\|\cdot\|\right.$ means $\left.\|\cdot\|_{L^{2}(\Omega)}\right)$

Let us summarize our numerical experiences. The computational results show the theoretical expected behavior. This concerns the dependence on $\varepsilon$ as well as the dependence on $\sigma_{c}$. The tests deliver numerical results in a range where the discretization error is small with respect to the investigated influences. 
Acknowledgement. We thank Klaus Krumbiegel for preparing the numerical tests. This research is supported by the FWF, Grant P18090-N12.

\section{References}

[1] Alt, W., On the approximation of infinite optimization problems with an application to optimal control problems. Appl. Math. Optim. 12 (1984)(1), $15-27$.

[2] Casas, E., Boundary control of semilinear elliptic equations with pointwise state constraints. SIAM J. Control Optim. 31 (1993)(4), 993 - 1006.

[3] Janno, J., Lavrent'ev regularization of ill-posed problems containing nonlinear near-to-monotone operators with application to autoconvolution equation. Inverse Problems 16 (2000)(2), 333 - 348.

[4] Lavrentiev, M. M., Some Improperly Posed Problems of the Mathematical Physics. New York: Springer 1967.

[5] Liu, F. and Nashed, M. Z., Convergence of regularized solutions of nonlinear illposed problems with monotone operators. In: Partial Differential Equations and Applications (ed.: P. Marcellini). Lecture Notes Pure Appl. Math. 177. New York: Marcel Dekker 1996, pp. 353 - 361.

[6] Meyer, C., Rösch, A. and Tröltzsch, F., Optimal control of PDEs with regularized pointwise state constraints. Comput. Optim. Appl. 33 (2006)(2-3), $209-228$.

[7] Nair, M., T. and Tautenhahn, U., Lavrentiev regularization for linear ill-posed problems under general source conditions. Z. Anal. Anwendungen 23 (2004)(1), $167-185$.

[8] Rösch, A. and Tröltzsch, F., Existence of regular Lagrange multipliers for elliptic optimal control problem with pointwise control-state constraints. SIAM J. Control Optim. 45 (2006)(2), $548-564$.

[9] Tautenhahn, U., On the method of Lavrentiev regularization for nonlinear illposed problems. Inverse Problems 18 (2002)(1), 191 - 207.

[10] Tröltzsch, F., Regular Lagrange multipliers for problems with pointwise mixed control-state constraints. SIAM J. Optim. 15 (2005)(2), $616-634$.

Received February 24, 2006; revised September 7, 2006 